\title{
New mechanism revealed for light-state transition in cyanobacterium Arthrospira platensis according to 77-K fluorescence kinetics
}

\author{
XU XiuLing ${ }^{\dagger}$, ZHANG Rui $^{\dagger}$, XIE Jie $^{*} \&$ ZHAO JingQuan* \\ Beijing National Laboratory for Molecular Sciences, Key Laboratory of Photochemistry, Institute of Chemistry, Chinese Academy of \\ Sciences, Beijing 100190, China
}

Received August 25, 2011; accepted November 5, 2011; published online February 23, 2012

\begin{abstract}
The mechanisms of oxygen evolution and carbon fixation in oxygenic organisms depend on the equal distribution of excitation energy to photosystems I and II, which is regulated by a mechanism referred to as light-state transition. In this work, a novel mechanism, energy spillover from PS I to PS II referred to as "inverse spillover", was revealed besides "mobile phycobilisome (PBS)" and the "spillover" of energy from PS II to PS I in cyanobacteria. Under continuous illumination with blue light, time-dependent 77-K fluorescence spectra demonstrated heterogeneous kinetics for the PBS and photosystem components, indicating that inverse spillover and mobile PBS work successively to regulate the excitation to a balanced distribution in cyanobacterial cells under blue light. Inverse spillover and mobile PBS occur under both 100 and $300 \mu \mathrm{mol} \mathrm{m}^{-2} \mathrm{~s}^{-1}$ blue-light conditions but they are accelerated under the latter.
\end{abstract}

cyanobacteria, light-state transition, blue light, 77-K fluorescence kinetics, mobile phycobilisome, inverse spillover

Citation: $\quad \mathrm{Xu} \mathrm{X} \mathrm{L,} \mathrm{Zhang} \mathrm{R,} \mathrm{Xie} \mathrm{J,} \mathrm{et} \mathrm{al.} \mathrm{New} \mathrm{mechanism} \mathrm{revealed} \mathrm{for} \mathrm{light-state} \mathrm{transition} \mathrm{in} \mathrm{cyanobacterium} \mathrm{Arthrospira} \mathrm{platensis} \mathrm{according} \mathrm{to} \mathrm{77-K} \mathrm{fluorescence}$ kinetics. Chin Sci Bull, 2012, 57: 1261-1265, doi: 10.1007/s11434-011-4962-5

Light-state transition is a function of oxygenic organisms to balance the excitations of photosystem I (PS I) and photosystem II (PS II) under variable light conditions and it is a necessary function for photosynthetic oxygen evolution and carbon fixation [1,2]. In green plants, state transition involves a directional movement of light-harvesting complex II to PS I or PS II $[3,4]$. In cyanobacteria, "mobile phycobilisome (PBS)" [1,5] or "energy spillover" from PS II to PS I [6-8] has been proposed to explain the state transition. Previously, using glycine betaine to immobilize PBSs on thylakoid membranes $[9,10]$, it was clarified that "mobile PBS" worked independently in light-induced state transitions while mobile PBS and energy spillover worked cooperatively in light-to-dark transition [11,12]. Light-state transitions in cyanobacteria were commonly studied in two discrete states, state 1 or state 2 , while the intermediate pro-

$\dagger$ These authors contributed equally to the work

* Corresponding authors (email: xiejie@iccas.ac.cn; zhaojq@iccas.ac.cn) cesses were of less concern. Previously, temporal fluctuation of the 77-K fluorescence for PBS rods, core, and PS I and PS II components was monitored when cyanobacterium Synechocystis PCC6803 cells were continuously illuminated with orange light, and the "damped-oscillation" fluorescence fluctuation revealed the dynamic behavior of PBSs to find a "balanced" position. Furthermore, it demonstrated that PBS and photosystem fluorescence components varied in homogeneous kinetics, indicating that PBSs regulated the excitation distribution independently [13]. While orange light is mainly absorbed by PBSs, it is understandable that a transition from state 1 to state 2 is completely regulated by mobile PBS. However, blue light is mainly absorbed by chlorophylls, and it is unreasonable that transition from state 2 to state 1 is regulated by mobile PBS only. In fact, whether a different mechanism is involved in transition from state 2 to state 1 is still unknown. In this work, 77-K fluorescence was monitored over time during blue-light- 
induced transition from state 2 to state 1 in cyanobacterium Arthrospira platensis cells. The heterogeneous kinetics of PBS and photosystem components, independent of the light intensity of 100 or $300 \mu \mathrm{mol} \mathrm{m} \mathrm{m}^{-2}$, indicated a novel mechanism, energy spillover from PS I to PS II referred to as "inverse spillover", involved in the state transition besides PBS mobility. Spectral analysis confirmed that inverse spillover was a natural mechanism and not an artificial result arising from spectral normalization.

\section{Materials and methods}

\subsection{Culture and growth conditions}

Arthrospira platensis FACHB-900 cells were cultured in $\mathrm{AB}$ medium ( $\mathrm{pH} 9.0,1 \mathrm{~L}$ ) at $25^{\circ} \mathrm{C}$ with continuous shaking and illumination by three $40-\mathrm{W}$ fluorescence lamps. Tenday cultures were harvested by centrifugation, washed and resuspended in fresh growth medium. Cells of the same generation were used in all experiments.

\subsection{State transitions}

For transition from state 2 to state 1 , the cells were previously induced to state 2 by orange light (Ditric Optics 580-nm long-pass and 600-nm short-pass filter) of $20 \mu \mathrm{mol}$ $\mathrm{m}^{-2} \mathrm{~s}^{-1}$ and were then immediately brought to state 1 by blue light (Ditric Optics 410-nm long-pass and 460-nm short-pass filter) of 100 or $300 \mu \mathrm{mol} \mathrm{m} \mathrm{m}^{-2}$. Afterward, the cells were immediately put into liquid nitrogen for measurement of 77-K fluorescence spectra. The light intensity was measured by an ultraviolet radiometer.

\subsection{Spectral measurements}

Fluorescence emission spectra at a temperature of $77 \mathrm{~K}$ were recorded by an F7000 spectrofluorometer (Hitachi,
Japan). The spectra presented in this work are the averages of five independent measurements. The excitation and emission slit widths were set to $5 \mathrm{~nm}$. The chlorophyll concentration of the cells used for fluorescence measurement was adjusted to no more than $5 \mu \mathrm{g}(\mathrm{Chl} a) \mathrm{mL}^{-1}$ estimated from the absorbance at $665 \mathrm{~nm}$ in methanol extracts [14].

\section{Results and discussion}

The 77-K fluorescence difference spectra were recorded over time when the state- 2 cells were continuously illuminated by blue light of $100 \mu \mathrm{mol} \mathrm{m} \mathrm{m}^{-2} \mathrm{~s}^{-1}$, as shown in Figure 1(a). From the spectra, the time-dependent fluorescence amplitudes for PBS rods (C-phycocyanin; C-PC), PBS core (allophycocyanin; APC) and PS I and PS II components were derived, as shown in Figure 1(b). A remarkable feature is that the PBS fluorescence components do not always keep pace with the fluorescence components of the photosystems, which completely differs from the case for orange-light-induced transition from state 1 to state 2 [13]. Figure 1(b) shows that the transition from state 2 to state 1 took $8 \mathrm{~min}$, at which time the PS I fluorescence and PS II fluorescence became saturated. However, the PBS components became invariable at $4 \mathrm{~min}$, at about half way into the transition to state 1 , suggesting that PBSs did not contribute further after that time. Apparently, the state transition undergoes two successive sub-processes. In the first $4 \mathrm{~min}$, the increase in C-PC and PS II fluorescence and the decrease in APC and PS I fluorescence suggests that PBSs move to PS II, which is a typical feature of transition from state 2 to state 1 [11,12], and the homogeneous kinetics of the PBS and photosystem fluorescence indicate that the state transition was regulated by mobile PBS. After $4 \mathrm{~min}$, PS II and PS I components increased and decreased respectively, independent of the PBS components, suggesting energy spillover from PS I to PS II, which is referred to as inverse spillover in this work.
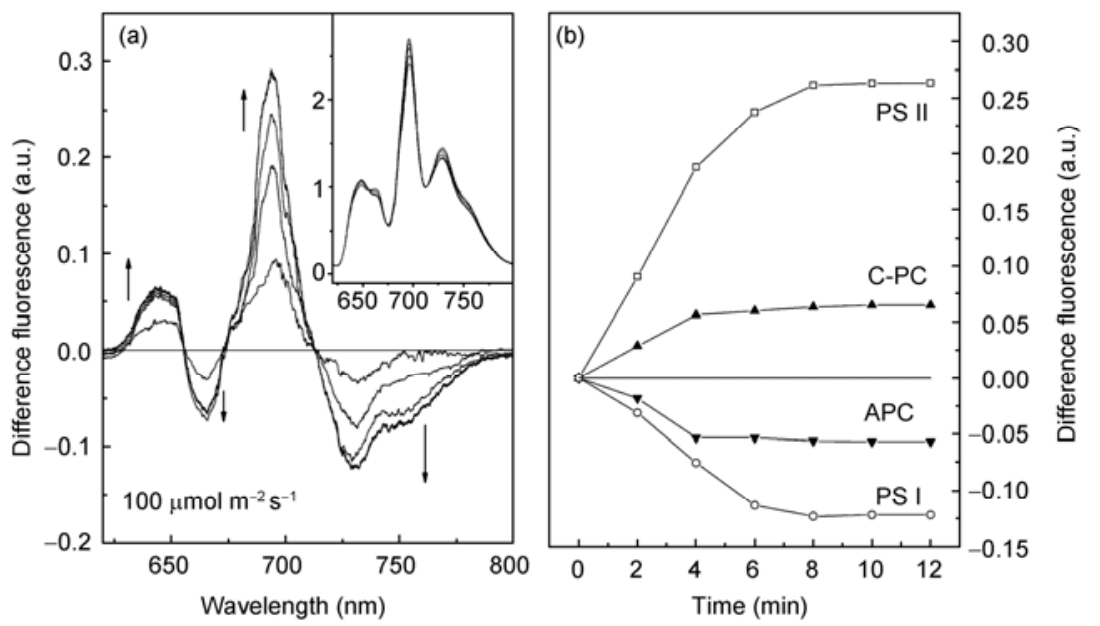

Figure 1 Difference fluorescence spectra (a) and plots of amplitudes of the four components against time (b) for state-2 cells illuminated by blue light at $100 \mu \mathrm{mol} \mathrm{m} \mathrm{m}^{-2}$, as derived from $77-\mathrm{K}$ fluorescence emission spectra (inset). Results are obtained for excitation at $580 \mathrm{~nm}$ and are normalized to $712 \mathrm{~nm}$. 
In the case that the cyanobacterium cells were illuminated by blue light of $300 \mu \mathrm{mol} \mathrm{m} \mathrm{m}^{-2} \mathrm{~s}^{-1}$, the time-dependent difference $77-\mathrm{K}$ fluorescence spectra and the component amplitudes are shown in Figure 2. The heterogeneous kinetics for PBS and photosystem components is similar to those in Figure 1. However, quantitatively, PS I and PS II fluorescence components became saturated at $2 \mathrm{~min}$ while the PBS components became saturated at $1 \mathrm{~min}$, suggesting that the state transition was accelerated compared with that under $100 \mu \mathrm{mol} \mathrm{m} \mathrm{m}^{-2} \mathrm{~s}^{-1}$ illumination. It was observed that the frequency of fluorescence oscillation or the state transition time for cyanobacterium Synechocystis PCC6803 cells was a function of orange-light intensity, which is ascribed to the acceleration of PBS movement at higher intensity [13]. The kinetics demonstrated in Figures 1 and 2 suggest that not only PBS movement but also inverse spillover was accelerated at higher light intensity, in turn suggesting that the state transition rate is a function of light intensity irrespective of whether a single mechanism or multiple mechanisms are involved. Importantly, this implies that cyanobacteria sense any imbalance of the excitation distribution and respond to it via state transition. Previously, it was proposed that state transition was a natural behavior of cyanobacteria under solar irradiation and not short-term acclimation under an artificial light condition [13], which is further confirmed by this work.

The fluorescence kinetics in Figures 1 and 2 suggest that mobile PBS and inverse spillover worked successively during the state transition. However, it might be argued that the heterogeneous kinetics were an artificial result of the normalization to $712 \mathrm{~nm}$ and not a natural behavior of the photo-functional entities. To clarify this, the spectra at 100 $\mu \mathrm{mol} \mathrm{m} \mathrm{m}^{-2} \mathrm{~s}^{-1}$ were alternatively normalized to several other wavelengths, for which the difference spectra and the time-dependent amplitudes are shown in Figure 3.

In Figure 3, the normalization wavelengths were selected quite arbitrarily for emission peaks (648 nm for C-PC or
$730 \mathrm{~nm}$ for PS I), a valley $(674.8 \mathrm{~nm})$ and a position between a peak and valley $(688 \mathrm{~nm})$. Noticeably, the fluorescence kinetics reproduce the basic feature in Figure 1, confirming that heterogeneous kinetics reveal a novel mechanism and are independent of normalization. Normalization is generally acceptable for $77-\mathrm{K}$ fluorescence spectra because it eliminates linearly correlated fluctuations and retains linearly independent "signals". In this sense, normalization to any wavelength in a spectrum is theoretically reasonable. However, normalization to a peak will conceal some components and amplify others, as shown in Figure 3(a)-(c). Consequently, normalization to a valley wavelength is a better choice.

Until now, it has been believed that mobile PBS and spillover of energy from PS II to PS I were involved in light-state transitions in cyanobacteria, whereas inverse spillover, the energy transfer from PS I to PS II, was revealed as an additive mechanism in the current work. In principle, light-state transition is a mechanism of interregulation between energetic and electronic states in oxygenic photosynthesis. At the molecular level, a complex mechanism is required to explain energy transfer from not only the photosynthetic antenna to the photosystems but also from one photosystem to another $[15,16]$ and electron transfer within PS II or PS I and between them. Specifically, under blue light, it is PS I that is overexcited while PS II is less excited; therefore, it is reasonable that there is energy spillover from PS I to PS II according to the energy-balance rule. Considering that the PS I energy level is lower than the PS II energy level, how the "uphill" transfer take place remains unknown. In fact, blue light resulting in overexcitation of PS I leads to excess oxidized reaction-center $\mathrm{P} 700^{+}$owing to a lack of PS II electrons, which may correlate to the inverse spillover in some way. It has been proposed that light-state transition in cyanobacteria might involve the movement of membrane complexes and/or changes in the oligomerization states of the complexes
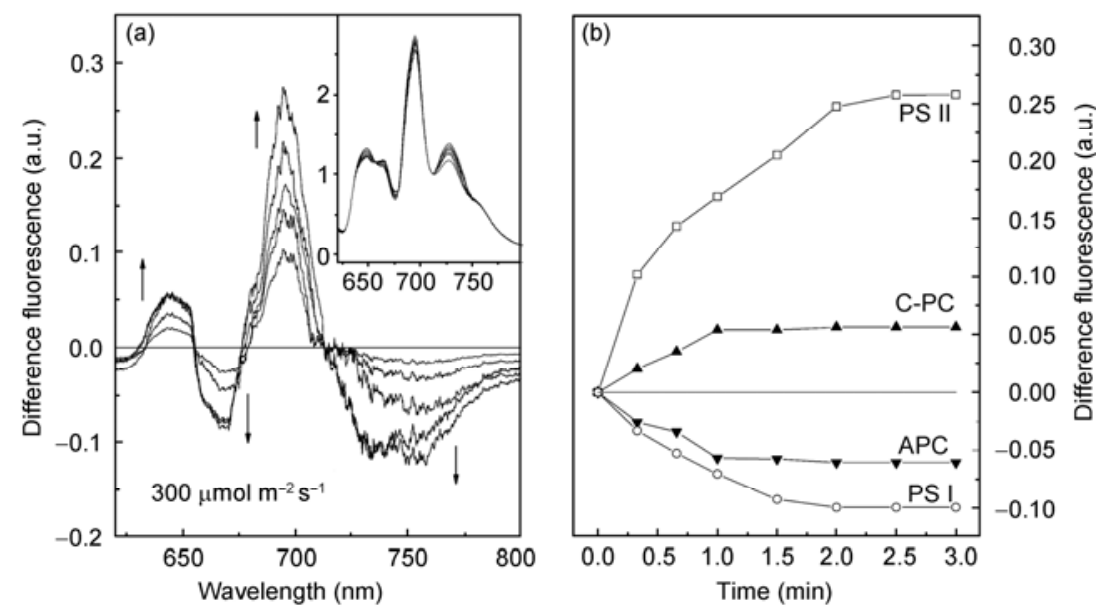

Figure 2 Difference fluorescence spectra (a) and plots of amplitudes of the four components against time (b) for state-2 cells illuminated by blue light of $300 \mu \mathrm{mol} \mathrm{m} \mathrm{s}^{-2}$, as derived from $77-\mathrm{K}$ fluorescence emission spectra (inset). Results are obtained for excitation at $580 \mathrm{~nm}$ and are normalized to $712 \mathrm{~nm}$. 

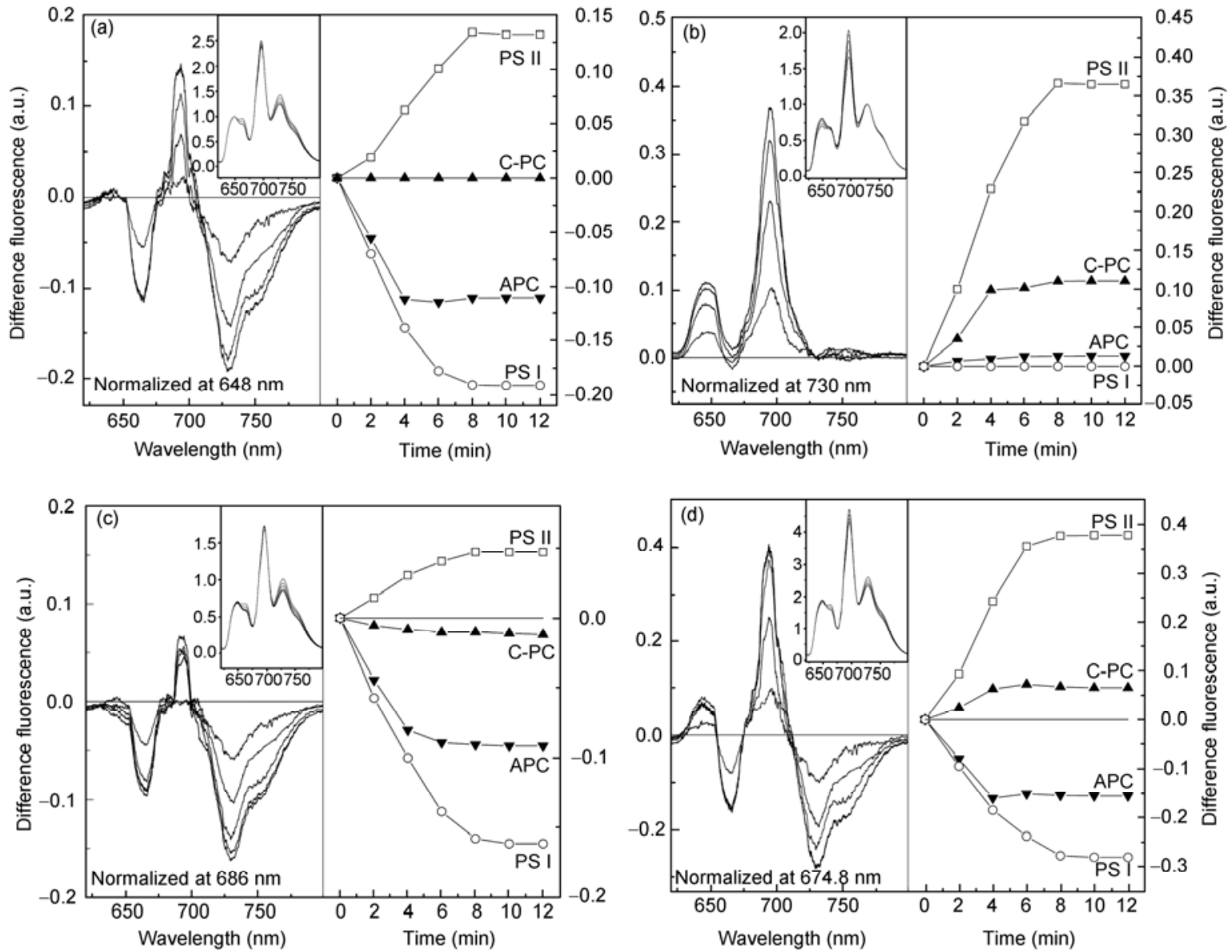

Figure 3 Difference fluorescence spectra (left panels) and plots of the amplitudes against time (right panels) derived from 77-K fluorescence emission spectra (insets, the same as Figure 1) normalized to $648 \mathrm{~nm}$ (a), $730 \mathrm{~nm}$ (b), $686 \mathrm{~nm}$ (c) and $674.8 \mathrm{~nm}$ (d).

(including PS II and PS I) [17,18]. It was found previously that PS I oligomerization states are reversibly variable under light-to-dark and dark-to-light transitions [12,19]. Apparently, either the movement of PS I or PS II or dissociation of PS I trimer into monomers may shorten the distance between PS I and PS II, amplify the effective concentration of PS I and enhance the spectral overlap of the PS I emission with PS II absorption, which certainly enhances the probability of uphill energy transfer. However, the molecular mechanism that triggers inverse spillover has yet to be revealed.

\section{Conclusion}

In the current work, a novel mechanism, energy spillover from PS I to PS II referred to as inverse spillover, was revealed for blue-light-induced state transition in cyanobacterium Arthrospira platensis FACHB-900 cells. Under blue light, 77-K fluorescence kinetics suggest that mobile PBS and inverse spillover worked successively and both were accelerated under a light condition of $300 \mu \mathrm{mol} \mathrm{m} \mathrm{m}^{-2} \mathrm{~s}^{-1}$ compared with those under a light condition of $100 \mu \mathrm{mol}$ $\mathrm{m}^{-2} \mathrm{~s}^{-1}$. The result suggests that cyanobacteria are able to balance the excitation distribution under various light conditions but the mechanisms involved may be multiple. Further research is necessary to clarify how inverse spillover occurs under blue light.

This work was supported by the National Natural Science Foundation of China (30970676).

1 Allen J F, Forsberg J. Molecular recognition in thylakoid structure and function. Trends Plant Sci, 2001, 6: 317-326

2 Bellafiore S, Barneche F, Peltier G, et al. State transitions and light adaptation require chloroplast thylakoid protein kinase STN7. Nature, 2005, 433: 892-895

3 Depege N, Bellafiore S, Rochaix J D. Role of chloroplast protein kinase Stt7 in LHCII phosphorylation and state transition in Chlamydomonas. Science, 2003, 299: 1572-1575

4 Allen J F. State transitions-A question of balance. Science, 2003, 299: $1530-1532$

5 Allen J F, Holmes N G. A general model for regulation of photosynthetic unit function by protein phosphorlation. FEBS Lett, 1986, 202: 175-181

6 Murata N. Control of excitation transfer in photosynthesis. I. Light-induced change of chlorophyll a fluorescence in Porphyridium cruentum. Biochim Biophys Acta, 1969, $172: 242-251$

7 Bruce D, Brimble S, Bryant D A. State transitions in a phycobili- 
some-less mutant of the cyanobacterium Synechococcus sp. PCC 7002. Biochim Biophys Acta, 1989, 974: 66-73

8 Federman S, Malkin S, Scherz A. Excitation energy transfer in aggregates of Photosystem I and Photosystem II of the cyanobacterium Synechocystis sp. PCC 6803: Can assembly of the pigment-protein complexes control the extent of spillover? Photosynth Res, 2000, 64: 199-207

9 Li Y, Zhang J, Xie J, et al. Temperature-induced decoupling of phycobilisomes from reaction centers. Biochim Biophys Acta, 2001, 1504: 229-234

10 Li D, Xie J, Zhao Y, et al. Probing connection of PBS with the photosystems in intact cells of Spirulina platensis by temperatureinduced fluorescence fluctuation. Biochim Biophys Acta, 2003, 1557: $35-40$

11 Li D, Xie J, Zhao J, et al. Light-induced excitation energy redistribution in Spirulina platensis cells: "Spillover" or "mobile PBSs"? Biochim Biophys Acta, 2004, 1608: 114-121

12 Li H, Li D, Yang S, et al. The state transition mechanism-Simply depending on light-on and -off in Spirulina platensis. Biochim Biophys Acta, 2006, 1757: 1512-1519

13 Yang S, Zhang R, Hu C, et al. The dynamic behavior of phycobilisome movement during light state transitions in cyanobacterium Synechocystis PCC6803. Photosynth Res, 2009, 99: 99-106
14 Porra R J, Thompson W A. Determination of accurate extinction coefficients and simultaneous equations for assaying chlorophylls $a$ and $b$ extracted with four different solvents; verification of the concentration of chlorophyll standards by absorption spectroscopy. Biochim Biophys Acta, 1989, 975: 384-394

15 Zhao G, Han K. Site-specific solvation of the photoexcited protochlorophyllide a in methanol: Formation of the hydrogen-bonded intermediate state induced by hydrogen-bond strengthening. Biophysical J, 2008, 94: 38-46

16 Zhao G, Han K. Site-selective photoinduced electron transfer from alcoholic solvents to the chromophore facilitated by hydrogen bonding: A new fluorescence quenching mechanism. J Phys Chem B, 2007, 111: 8940-8945

17 Bald D. Supramolecular architecture of cyanobacterial thylakoid membranes: How is the phycobilisome connected with the photosystems? Photosynth Res, 1996, 49: 103-118

18 Kaňa R, Prášil O, Komárek O. GC papageorgiou, govindjee. Spectral characteristic of fluorescence induction in a model cyanobacterium Synechococcus sp. (PCC 7942). Biochim Biophys Acta, 2009, 1787: $1170-1178$

19 Zhang R, Xie J, Hu C, et al. Hydrogen ions could directly regulate the oligomerization state of photosystem I in intact Spirulina platensis cells. Chin Sci Bull, 2008, 53: 317-320

Open Access This article is distributed under the terms of the Creative Commons Attribution License which permits any use, distribution, and reproduction in any medium, provided the original author(s) and source are credited. 